\title{
La integración del colectivo inmigrante en las regiones españolas
}

\author{
Antidio Martínez de Lizarrondo Artola \\ Gobierno de Navarra (Observatorio de la Realidad Social) \\ Universidad Pública de Navarra \\ amartart@navarra.es; antidio.martinez@unavarra.es

\section{Sebastian Rinken} \\ Instituto de Estudios Sociales Avanzados (IESA-CSIC) \\ srinken@iesa.csic.es

\section{Gorka Moreno Márquez} \\ Universidad del País Vasco y Observatorio Vasco de Inmigración (Ikuspegi) \\ gorka.moreno@ehu.eus

\section{Dirk Godenau} \\ Universidad de La Laguna y Observatorio de la Inmigración de Tenerife (OBITen) \\ dgodenau@ull.es
}

\section{Resumen}

Este artículo presenta un novedoso sistema de medición de la integración de las personas inmigrantes en las regiones españolas. Complementando en varios aspectos la iniciativa lanzada años atrás por las instituciones europeas (Declaración de Zaragoza), el sistema de medición propuesto comprende 24 indicadores que abarcan cuatro áreas: la situación laboral; las relaciones sociales e intergrupales; el bienestar individual, y el acceso a derechos de ciudadanía. Basándose en un amplio abanico de fuentes secundarias, consigue algo inédito: una completa cobertura estadística a escala infraestatal. El sistema sienta las bases para un seguimiento longitudinal y su ejecución con datos de 2011 permite comprobar el impacto de los primeros años de crisis económica sobre la integración de la población inmigrante. Los resultados señalan cierta ambivalencia, con una situación crecientemente desfavorecida de las personas inmigrantes en los ámbitos de Empleo y Bienestar, en comparación con la población nativa. En cambio, en los ámbitos de Ciudadanía y Relaciones Sociales se observa una evolución positiva, incluso en el periodo de recesión económica. La naturaleza polifacética de estos procesos y las disparidades territoriales dan lugar a tres perfiles regionales distintos.

Palabras clave: integración; inmigración; cohesión social; crisis económica; diversidad cultural; indicadores; bienestar 
Abstract. The integration of the immigrants in Spain and its regions

This paper presents an innovative measurement system regarding the integration of immigrants in Spain and its regions. Complementing in various ways the EU-sponsored Declaration of Zaragoza, the approach proposed here includes 24 indicators grouped into four areas: employment, social and intergroup relations, individual welfare, and citizenship. Based on a wide range of secondary sources, it is the first measurement system of immigrant integration that achieves comprehensive statistical coverage at the infra-national level. Its indicators allow for longitudinal measurement; by comparing 2007 and 2011 data, the impact of the ongoing economic crisis' initial years on the integration of immigrants is discerned. The results evidence some ambivalence: immigrants experience increasing disadvantages in the domains of employment and welfare compared with Spaniards; in contrast, in the areas of citizenship and social relations, integration has evolved favorably even in the context of economic recession. Multifaceted integration processes and territorial disparities originate three separate regional profiles.

Keywords: integration; immigration; social cohesion; economic crisis; cultural diversity; indicators; welfare

\author{
Sumario \\ Introducción 3. Resultados \\ 1. Conceptualización y medición del 4. Conclusiones \\ proceso de integración \\ 2. Datos y metodología Anexo
}

\title{
Introducción
}

En estos últimos años, la medición de la integración social y económica del colectivo inmigrante se ha convertido en uno de los temas prioritarios de los estudios sobre las migraciones internacionales que son impulsados por las instituciones competentes. De manera destacada, la Unión Europea ha estado estimulando el desarrollo de propuestas de medición estadística de los procesos de integración, con el objetivo de comparar entre distintos países y, en su caso, identificar tendencias comunes. Dos de los retos básicos a los que se enfrentan tales intentos son, por un lado, la dificultad para captar la complejidad de los procesos de integración, notoriamente multifacéticos, y, a veces, escurridizos en cuanto a su medición, y, por otro, la obtención de datos comparables y representativos para todos los territorios en cuestión. Esta última dificultad se acentúa cuando se pretende comprobar hasta qué punto el desarrollo de dichos procesos difiere a escala infraestatal; interrogante este que resulta especialmente pertinente en países que se caracterizan por marcadas diferencias entre sus regiones, como es el caso de España.

En este artículo, presentamos un novedoso sistema de medición de la integración de las personas inmigrantes en las regiones españolas, elaborado por 
un equipo formado por miembros de cuatro observatorios de la inmigración ${ }^{1}$ y que cubre aspectos de importancia innegable, pero de difícil determinación mediante herramientas estadísticas, como son las relaciones sociales. El sistema de indicadores comprende un índice sintético de integración y permite realizar un seguimiento longitudinal, no precisa de nuevas operaciones estadísticas, sino que aprovecha fuentes oficiales ya existentes. Con relación a cada uno de los cuatro ámbitos discernidos, la situación de las personas de procedencia extranjera es comparada con la observada para la población española. La equiparación entre ambas está definida como integración.

En la versión que se presenta aquí, elaborada con datos de 2011, el sistema de medición permite calibrar los efectos del primer periodo de la reciente crisis para las poblaciones autóctona y alóctona. Entre los resultados, cabe destacar que los patrones regionales en los distintos ámbitos carecen de un denominador único. Puede subrayarse que las diferencias con respecto a la media nacional en un determinado ámbito tienden a compensarse con diferencias en sentido contrario en otro. Así pues, pueden observarse distintas tipologías en cuanto a la combinación de fortalezas y debilidades relativas.

El artículo se divide en cuatro epígrafes. En el primero, se contextualiza el concepto de integración y se exponen los antecedentes que han guiado la elaboración de la propuesta de medición. A continuación, se detallan las bases conceptuales y metodológicas para concretar los ejes y seleccionar los indicadores, así como las técnicas utilizadas para tipificar los perfiles a escala regional. Seguidamente, se procede a la exposición sinóptica de los resultados, tanto para cada uno de los cuatro ámbitos de medición, como para toda la información obtenida en su conjunto, siempre con el fin de establecer perfiles regionales. El artículo finaliza con un resumen de las principales conclusiones.

\section{Conceptualización y medición del proceso de integración}

La integración del colectivo inmigrante es un objetivo destacado para muchas sociedades receptoras. Del mismo modo, su medición se ha convertido en uno de los ejes de actuación dentro de sus políticas migratorias, para así tener criterios mucho más sólidos y estables de cara a la intervención pública y social. En este sentido, la integración condiciona e influye en la dinámica social general y en la cohesión social de la colectividad, entendida esta como un proceso que incluye diferentes dimensiones y una multitud de factores. El propio concepto de integración no deja de ser escurridizo en cuanto a su significado. Este hecho nos lleva frecuentemente a la necesidad de la pulcritud estadística para su medición, puesto que esta requiere herramientas adecuadas y precisas que

1. Este artículo se basa en una investigación patrocinada por la Secretaría de Estado de Inmigración y Emigración (Godenau et al. [coords.], 2014), que ha sido realizada por cuatro observatorios de la inmigración. Estos organismos son el Observatorio Permanente de la Inmigración en Navarra (OPINA), el Observatorio de la Inmigración de Tenerife (OBITen), el Observatorio Permanente Andaluz de las Migraciones (OPAM) y el Observatorio Vasco de Inmigración (Ikuspegi). 
reconozcan su carácter multidimensional y su necesaria ubicación contextual. $\mathrm{Al}$ respecto, no puede dejarse a un lado que, sea cual sea la óptica desde la que se estudien las concepciones sobre la integración, nos ofrecen una visión de la sociedad y del tipo de relaciones y de dinámicas que en ella tienen lugar. La historia nos muestra ejemplos de esta afirmación, y así, desde los tiempos de la Escuela de Chicago (Thomas y Znaniecki, 1996), se han estudiado las dimensiones de la integración que influyen en la inserción de personas de origen diverso en una sociedad. Por ello, es imprescindible averiguar los ritmos, las dimensiones y el sentido de dichos procesos.

El término integración es usado con profusión en la literatura sobre las migraciones internacionales, si bien bajo un elevado grado de indeterminación (Spencer, 2006). A menudo, se efectúan reducciones culturalistas y se obvian argumentaciones basadas en las lógicas de funcionamiento social y económico de la propia sociedad. Esto es, las pautas culturales no marcan todo el largo proceso que implica la integración en una sociedad ajena. Hammar y Lithman (1989) aducen que hay tantos significados de integración como personas tratan el tema de las relaciones entre inmigrantes y autóctonos. Como idea general, podemos asumir que el asentamiento de personas inmigrantes conlleva su interacción con autóctonas, una creciente familiaridad con las costumbres y las instituciones de la sociedad de acogida y, además, una serie de cambios que implican a ambas, aunque quizás en distinta medida e intensidad (Schierup y Alund, 1986).

La construcción teórica de tres modelos (asimilacionismo, crisol y pluralismo cultural) ayuda a avanzar en la precisión terminológica de este concepto, cuanto menos en su vertiente más culturalista. Según el primer modelo (Gordon, 1964; Alba y Nee, 1997), las minorías inmigradas se irían desprendiendo de su cultura para adoptar las formas de vida y las costumbres mayoritarias de la sociedad de destino, pero Joppke y Morawska (2003) critican que se parta de una concepción estática y homogénea de la sociedad de acogida que no se corresponde con la realidad. Por su parte, en el crisol, la identidad nacional se configura a partir de las contribuciones de todos los grupos poblacionales (Gordon, 1975), aunque Lambert y Taylor (1988) y Giménez (1996) ven aquí una impronta del asimilacionismo. El pluralismo es el tercer paradigma, que supone que es posible la convivencia armoniosa en sociedades diversas enfatizando la heterogeneidad cultural (Blanco, 2000). La multiculturalidad y la interculturalidad representan este modelo. Ambas remiten a la coexistencia de grupos con culturas diferentes en un mismo espacio, pero la primera es utilizada más como sinónimo de pluralidad (Aguado, 1997) y la segunda incide en la interrelación entre culturas y en el logro de una convivencia basada en la igualdad, la no discriminación y el respeto a la diversidad (Rodríguez Rojo, 2006). En la actualidad, las tesis pluralistas de gestión de la diversidad han retrocedido y se van intensificando las demandas unidireccionales de integración dirigidas a la población inmigrante (Vertovec, 2010).

Aparte de la tendencia a mezclar el análisis empírico con expectativas normativas, estos tres modelos se exponen a una serie de críticas de calado. Entre 
ellas, cabe destacar que se identifica la pluralidad de las sociedades receptoras con la llegada de población inmigrante, como si estas sociedades fueran monolíticas y sin diversidad con anterioridad (Richmond, 1988; Lamo de Espinosa, 1995). Asimismo, se le da demasiada relevancia al ámbito cultural, lo que puede conducir a un diferencialismo que puede servir de base para el fundamentalismo racista (Brah, 1996; Provansal, 1999). Pese a que la escala regional y local es el principal escenario de la integración (Penninx et al., 2004), estos modelos genéricos evidencian una falta de atención hacia este nivel infraestatal y una lógica analítica en la que prima la dimensión estatal como objeto de estudio (Lahav, 1998). Por último, la integración no es progresiva y lineal, sino más bien un proceso abierto e influenciado por las variables tiempo y espacio, lo que denominamos proyecto migratorio (Dassetto, 1990), en el que muchos avances son reversibles o pueden tener un carácter regresivo (Telles y Ortiz, 2011).

Pasando a un plano más aplicado del concepto, hay que destacar que, frecuentemente, se observa una ausencia de estrategias de integración coherentes en todos los ámbitos de la realidad (Engelen, 2003). Esta falta de planificación es evidente en España, ya en los primeros años noventa, por cuanto no existía un plan previo para gestionar los flujos de llegada (Arango, 1999) y la activación del mercado de trabajo como principal vector de integración (Izquierdo y León-Alfonso, 2008). Este hecho ha devenido en una serie de debilidades y carencias dentro del proceso de integración del colectivo inmigrante. En primer lugar, la incorporación de personas inmigrantes se ha producido en el marco de un amplio abanico de normas e instituciones prediseñadas con fines ajenos al hecho inmigratorio (Soysal, 1994). En segundo lugar, se omiten los efectos potencialmente contradictorios de las prácticas vigentes, esto es, las políticas incluyentes con respecto a las personas inmigrantes pueden constituir la compensación de una política de inmigración excluyente (Joppke,1999; Guiraudon, 2000).

Así pues, los modelos de integración utilizados para describir las formas de incorporación de estas poblaciones en nuestras sociedades han recibido críticas relativas a dosis excesivas de determinismo y de esencialismo. A partir de esta constatación, es preciso resaltar el esfuerzo académico por superar la incómoda coexistencia de intentos de descripción, por un lado, y de propuestas normativas, por otro. Entre las proposiciones más solventes, resaltan la teoría de la asimilación segmentada (Portes y Böröcz, 1989), la perspectiva desagregada (Freeman, 2004) y la noción de integración intercultural (Bosswick y Heckmann, 2006). Sus rasgos comunes son el interés por buscar diagnósticos matizados y que en ningún caso se aplica una visión finalista de la integración. La cuestión no es conseguir que se produzca integración en línea con un ideal determinado, sino constatar cómo se desarrollan estos procesos en distintos ámbitos de la realidad. En ello desempeñan un papel central la estructura social y las instituciones que conforman la sociedad receptora.

En esta cuestión, es preciso resaltar la importancia de la vertiente regional. La gran heterogeneidad existente en materia de inmigración e integración 
repercute en el hecho que las diferencias entre las regiones de un país puedan ser notables. Algunos de los factores que explican esta diversidad son los niveles de descentralización político-administrativa, las características socioeconómicas y las particularidades de los flujos migratorios en cada región (Phalet y Swyngendouw, 2003; Rodríguez-Izquierdo, 2013; Huddleston et al., 2013). $\mathrm{La}$ influencia de estos factores en el Estado español ha sido frecuentemente constatada, de modo que ha favorecido la manifestación de distintas trayectorias de "mosaicos migratorios» (Cachón, 2008) o el denominado «modelo patchwork» de integración (Martínez de Lizarrondo, 2009). Teniendo en cuenta estos aspectos, se estima que un sistema de medición a escala regional capaz de captar la complejidad de estos procesos es un avance muy relevante para profundizar en este ámbito.

Muchos científicos sociales e instituciones han planteado en los últimos años la idoneidad de poseer sistemas específicos de medición de los procesos de integración de las poblaciones inmigrante y autóctona en nuestras sociedades. Los antecedentes teóricos muestran la relevancia que han tenido en este campo ciertas dimensiones, así como la habitual dificultad para obtener datos sobre indicadores que atiendan estos procesos. García Cívico (2011) señala estos trabajos como precursores en el estudio de la integración con indicadores, al aportar valiosas herramientas de análisis y comparación. Por ejemplo, conviene recordar el Proyecto I3 (Ministerio de Trabajo y Asuntos Sociales, 2007), con premisas conceptuales y metodológicas semejantes a nuestra propuesta, al basarse en fuentes secundarias para armonizar los indicadores elegidos. Otra idea sugerente para medir múltiples dimensiones es el barómetro social de España (Colectivo Ioé, 2008), si bien aborda la realidad migratoria tangencialmente. También en términos generales es significativo el sistema de indicadores de la Fundación FOESSA, que plantea el VI Informe sobre exclusión y desarrollo social en España (Laparra y Pérez, 2008).

Dentro del ámbito institucional, es especialmente relevante para nuestro trabajo la Declaración de Zaragoza, de la Conferencia Ministerial Europea sobre Integración (2010). Esta propuesta establece cuatro dimensiones para medir el concepto de integración: empleo, educación, inclusión social y ciudadanía activa. Si bien es incompleto en cuanto a las facetas de la realidad social que abarca, aporta un aspecto clave: el requisito de abastecer los indicadores a partir de fuentes secundarias, lo que dota al sistema de viabilidad y periodicidad. Eurostat (2011) puso en práctica las intenciones de Zaragoza a través de un informe que proporciona cifras comparables de los indicadores básicos contemplados para los países de la UE. En una línea similar, aparece el estudio desarrollado por la OECD (2012), que agrupa 21 indicadores en siete dimensiones. Más recientemente, la Comisión Europea publicó un informe de Huddleston et al. (2013), que refleja que varios países de la Unión Europea ya han establecido indicadores de integración y sistemas de medición de carácter nacional. Por último, el III MIPEX - Migrant Integration Policy Index- (Huddleston et al., 2011) supone otro enfoque que se centra en el análisis del marco normativo relativo a la integración. Todos estos intentos raramente han tenido en 
cuenta el nivel regional, y ello a pesar de que el Comité de las Regiones (2012) ha manifestado su intención de contribuir a la creación de un sistema común de indicadores para medir los progresos realizados en materia de integración.

A partir de los antecedentes citados y de la conceptualización ampliada y comprensiva del objeto de estudio, se ha conformado nuestro sistema de medición, que se compone de cuatro ámbitos y que coincide en gran parte con la estructura de la Declaración de Zaragoza. En concreto, son Empleo, Bienestar, Ciudadanía y Relaciones Sociales e Intergrupales. Este último es precisamente el que difiere con respecto al antecedente citado, ya que se ha estimado que esta dimensión, dentro de la medición de la integración, era necesaria y tenían que incluirse indicadores asociados a las relaciones sociales. De igual forma, hemos unido en una misma categoría las dimensiones Bienestar, Educación e Inclusión Social de la Declaración de Zaragoza.

\section{Datos y metodología}

Los problemas metodológicos que plantea un análisis de la integración de las personas inmigrantes a escala regional son múltiples. Con carácter previo y tras la revisión sistematizada de los antecedentes existentes, se establecieron las dimensiones de medición que dan forma a nuestro sistema, optando por aquellas que generan un amplio consenso en torno a la relevancia que tienen en el proceso de integración de las personas inmigrantes. En el caso del empleo, se trata de un elemento básico para la vertebración social en las sociedades modernas. A su vez, es un factor clave para la definición de la posición social de cualquier persona, ya sea esta inmigrante o autóctona. Un adecuado nivel de bienestar es también fundamental para la integración y, al mismo tiempo, se convierte en resultado de dicho proceso. En el caso de las relaciones sociales, más allá de no ser habitualmente un espacio para la intervención pública, estas son de vital importancia en cualquier proceso de integración, tal y como ha podido verse en las últimas décadas a través de la constatación de las distancias existentes entre población autóctona e inmigrante. Por último, cabe destacar que la ciudadanía se convierte, en muchas ocasiones, en un elemento previo y en una puerta de acceso para el disfrute de derechos políticos, civiles y sociales. Teniendo en cuenta estas cuestiones, estimamos que estas dimensiones $-\mathrm{y}$ la relación entre ellas - compone un marco sólido y estable para poder medir la integración del colectivo inmigrante de forma fiable y satisfactoria.

Paso seguido, en cada una de estas áreas, se han establecido una serie de criterios para garantizar la viabilidad y la fiabilidad de los indicadores. Este paso es igualmente crucial, ya que representa la transición, o la conexión, entre conceptualización y mensurabilidad. Conexión que muchas veces no corresponde al diseño ideal del investigador, puesto que la mensurabilidad se ve restringida, a efectos prácticos, por la disponibilidad de datos. Entre los criterios seleccionados, se encuentran la disponibilidad de una muestra suficiente para la elaboración del indicador; la procedencia del dato de una fuente estadística oficial o, por lo menos, bien consolidada; la periodicidad del dato; 
la posibilidad de desagregación según nacionalidad; la posible diferenciación entre nacionales de países comunitarios y no comunitarios; la opción de poder desagregar el dato según la escala infraestatal, y la garantía de que la interpretación del dato es clara y evita ambigüedades.

En consecuencia, se han desechado las fuentes de información primarias por su elevado coste, porque exigirían muchos esfuerzos para garantizar su fiabilidad y validez y, además, porque añadirían incertidumbre en el mantenimiento futuro del sistema. Las fuentes elegidas son secundarias, porque contribuyen a la sostenibilidad en el tiempo del sistema de indicadores. Las trece fuentes estadísticas finalmente seleccionadas y su correspondencia con cada indicador se exponen en la tabla 4 (anexo).

A continuación, y para la construcción de indicadores, los valores obtenidos en cada punto de medición se convierten, en todos los casos, en una relación entre dos magnitudes, esto es, en cocientes. Esta fórmula permite comparar la situación de la población extranjera con la española. Entendemos que esta opción es preferible a la comparación entre extranjeros y el total de la población, que es la propuesta de la Declaración de Zaragoza, ya que así evitamos que la población evaluada esté incluida en el conjunto del total. Asimismo, admite fácilmente la comparabilidad interregional. En algunos casos, por las características del indicador, no ha sido posible poner en relación ambas poblaciones. Ante estas situaciones, se ha optado por incluir tasas o proporciones en el indicador. La construcción del cociente es variable en función de la naturaleza de los datos, es decir, se relacionan tasas de extranjeros y españoles, alternando su posición en el numerador y en el denominador para que la interpretación final sea siempre homogénea y más sencilla, de tal modo que valores por encima de 1 sean siempre situaciones favorables a la población española y valores por debajo configuren un escenario más favorable para el colectivo extranjero. El valor 1 es la referencia igualitaria.

De esta forma, cuanto más elevado sea un cociente, mayor será la desigualdad de los extranjeros; y a la inversa, en la medida en que más se acerque a 1 , señalará mayor igualdad e integración entre ambos colectivos. Por lo tanto, se ha partido de una concepción de la integración definida como equiparación, siendo conscientes de las objeciones que puede tener esta acepción, como, por ejemplo, la integración a la baja, es decir, la posibilidad de que una región muestre una mejor integración —un valor más cercano a 1 - a costa de una peor situación, tanto del colectivo inmigrante como del autóctono. Sin embargo, posee la virtud de facilitar la comparabilidad, tanto intrarregional como interregional, así como de ofrecer una herramienta operativa de medición fácilmente comprensible.

Con todo, los indicadores que forman el sistema transversal de medición son veinticuatro: siete en Empleo (Actividad, Empleo, Paro, Temporalidad, Ocupaciones elementales, Sobrecualificación y Ganancia media), cinco en Bienestar (Hogares que llegan con dificultad a fin de mes, Gasto medio del hogar, Personas por habitación, Estado de salud y Estudios superiores), cinco en Relaciones sociales e intergrupales (Hogares mixtos, Convivencia de parejas 
en el hogar, Hogares con menores dependientes, Capacidad lingüística y Aceptación) y siete en Ciudadanía (Regularidad documental, Residencia de larga duración, Nacionalización por residencia, Sufragio activo, Sufragio pasivo, Escolaridad infantil y Escolaridad postobligatoria).

$\mathrm{Al}$ igual que en el caso de las dimensiones, para la selección de los indicadores, se ha realizado una labor de revisión bibliográfica, así como de reflexión interna, de cara a seleccionar en cada ámbito los indicadores que nos aportan mayor información con respecto a los procesos de integración de la población inmigrante.

Obviamente, hubo indicadores que bien podían haber formado parte del sistema, pero se descartaron por falta de datos (de fuentes secundarias adecuadas, de informaciones a escala regional o desagregados por nacionalidad) y por ambigüedad (porque tienen una doble lectura, positiva y negativa, de los procesos de integración). Aquí constan indicadores que quizás hubieran complementado mejor áreas que vislumbran dificultades para su medición completa, como Relaciones sociales o Ciudadanía. Es el caso de algunos indicadores que midieran las redes sociales y las interacciones interpersonales voluntarias, o el sentimiento de pertenencia, el acceso a la sanidad, la percepción de discriminación y la movilidad social, respectivamente.

Otra limitación del sistema es que, dadas las fuentes, la mayoría de indicadores no incluye a las personas naturalizadas y se centran en la dicotomía entre extranjero y español. Además, por la imposibilidad de disponer de datos representativos a nivel autonómico, la aproximación a la diversidad regional se realiza mediante las siete agrupaciones NUTS-1 de Eurostat, que corresponden a agrupaciones de comunidades autónomas en regiones: Noroeste (Galicia, Asturias y Cantabria), Nordeste (País Vasco, Navarra, La Rioja y Aragón), Comunidad de Madrid, Centro (Castilla y León, Castilla-La Mancha y Extremadura), Este (Cataluña, Comunidad Valenciana y Baleares), Sur (Andalucía, Región de Murcia y las ciudades de Ceuta y Melilla) y Canarias. Aquí conviene subrayar que son territorios amplios que se caracterizan, a veces, por una pronunciada heterogeneidad interna y que, además, tampoco coinciden con zonas funcionales (urbanas y rurales, y turísticas, agrarias e industriales), por lo que la media regional puede componerse de datos relativos a zonas muy diferentes entre sí.

Finalmente, a partir de esos datos, se realizó una búsqueda de patrones geográficos a través de la elaboración de índices sintéticos (resultado de medias geométricas ponderadas por análisis discriminante) y la clasificación regional por medio del análisis clúster (elaborado a través de técnicas multivariantes), para la diferenciación de patrones regionales. Los índices sintéticos representan el intento de resumir en un único valor numérico el grado de integración de las personas inmigrantes con respecto a la población española en cada región y en cada dimensión analítica. Por lo tanto, se obtuvieron cinco índices sintéticos para cada una de las siete regiones NUTS-1, uno por dimensión, más otro general que abarca todos los ejes. Sin obviar que tal síntesis conlleva una pérdida de especificidad y que su interpretación puede ser complicada, su 
utilidad reside precisamente en la posibilidad de poder resumir un amplio abanico de datos en un solo valor interpretable en el sentido de una mejor o peor integración.

\section{Resultados}

En este apartado, se exponen, en primer lugar, los principales resultados de las cuatro dimensiones analizadas en nuestra investigación, subrayando las diferencias existentes entre personas españolas y extranjeras. Posteriormente, en el siguiente epígrafe, analizaremos las posibles diferencias regionales en la integración del colectivo inmigrante y plantearemos la existencia o no de perfiles regionales de integración.

\subsection{Resultados por dimensión}

A continuación, presentaremos los datos generales de cada dimensión a nivel estatal y a escala territorial NUTS-1, incidiendo en los valores de los cocientes de cada indicador para el año 2011. Estos valores se exponen en la siguiente tabla, que sirve para observar las diferencias y las similitudes regionales a través de colores (sombreado verde, blanco y rojo) que reflejan los niveles más positivos, intermedios y desfavorables, respectivamente. De cara a una mejor comprensión de la tabla, es importante destacar que las magnitudes de cada uno de los cocientes no pueden ser comparadas entre indicadores.

En esta tabla, se observa que, de los siete indicadores en el ámbito del Empleo, cinco obtienen puntuaciones superiores a 1 en el conjunto de España (última columna), lo que es indicativo de una peor situación de los extranjeros. Entre estos cinco, destacan la sobrecualificación, la concentración en ocupaciones elementales y la contratación temporal por sus elevados valores, es decir, aspectos en los que las diferencias entre extranjeros y nacionales son máximas. Los dos aspectos que indican situaciones favorables son la actividad y el empleo, siendo las elevadas tasas de actividad de la población extranjera la causa de sus tasas de empleo superior, pese a las, también elevadas, tasas de paro. En definitiva, nos encontramos ante una mayor precarización laboral por parte del colectivo extranjero, unido a una mayor tasa de actividad, que se explica, en gran parte, por una estructura de edad mucho más joven que la población española.

Las desviaciones regionales de la media nacional en cada indicador son de amplitudes heterogéneas: mayores en paro, en ocupaciones elementales y en sobrecualificación; y menores en actividad y empleo, en contratación temporal y en ganancias salariales. El patrón que se detecta en la tabla sinóptica permite generalizar sobre las diferencias entre tipos de regiones en materia de empleo:

1. Sur y Canarias, donde las diferencias entre extranjeros y nacionales son menores que en el conjunto del país, pero en un contexto general negativo en términos socioeconómicos. 
Tabla 1. Valores de los indicadores de integración por regiones NUTS-1 en 2011

\begin{tabular}{|c|c|c|c|c|c|c|c|c|}
\hline Indicador & Noroeste & Nordeste & Madrid & Centro & Este & Sur & Canarias & España \\
\hline E1 & 0,71 & 0,71 & 0,73 & 0,69 & 0,81 & 0,80 & 0,82 & 0.76 \\
\hline E2 & 0,91 & 0,91 & 0,83 & 0,84 & 1,02 & 0,92 & 0,87 & 0,91 \\
\hline E3 & 2,16 & 2,67 & 1,69 & 1,75 & 1,93 & 1,34 & 1,14 & 1,68 \\
\hline E4 & 2,13 & 2,07 & 2,12 & 1,84 & 2,08 & 1,83 & 1,79 & 1,90 \\
\hline E5 & 3,80 & 4,40 & 4,13 & 3,25 & 3,30 & 3,46 & 1,95 & 3,36 \\
\hline E6 & 6,37 & 8,24 & 7,66 & 8,36 & 7,61 & 8,07 & 4,21 & 7,24 \\
\hline E7 & 1,35 & 1,46 & 1,58 & 1,42 & 1,44 & 1,44 & 1,47 & 1,49 \\
\hline B1 & 1,75 & 3,13 & 1,80 & 1,43 & 1,82 & 1,53 & 1,38 & 1,73 \\
\hline B2 & 1,37 & 1,54 & 1,34 & 1,33 & 1,34 & 1,22 & 1,17 & 1,30 \\
\hline B3 & 1,39 & 1,56 & 1,56 & 1,24 & 1,29 & 1,35 & 1,09 & 1,38 \\
\hline B4 & 1,07 & 1,13 & 1,09 & 1,05 & 1,02 & 1,01 & 1,17 & 1,04 \\
\hline B5 & 1,63 & 1,48 & 1,25 & 1,64 & 1,08 & 0,99 & 0,83 & 1,11 \\
\hline R1 & 1,90 & 2,77 & 3,04 & 2,96 & 3,11 & 3,17 & 2,24 & 2,92 \\
\hline R2 & 1,06 & 1,03 & 1,03 & 1,01 & 1,00 & 1,00 & 1,03 & 1,01 \\
\hline R3 & 0,47 & 0,54 & 0,55 & 0,48 & 0,65 & 0,86 & 0,78 & 0,64 \\
\hline R4 & 1,89 & 1,85 & 1,57 & 2,70 & 2,22 & 2,51 & 1,69 & 2,03 \\
\hline R5 & 1,40 & 1,18 & 1,15 & 1,25 & 1,28 & 1,29 & 1,25 & 1,26 \\
\hline C1 & 1,13 & 1,09 & 1,24 & 1,00 & 1,13 & 1,10 & 1,05 & 1,13 \\
\hline C2 & 1,99 & 1,58 & 1,55 & 1,52 & 1,43 & 1,39 & 1,50 & 1,48 \\
\hline C3 & 7,93 & 9,33 & 6,57 & 13,03 & 11,51 & 15,85 & 11,10 & 10,20 \\
\hline C4 & 8,66 & 7,47 & 7,12 & 6,08 & 6,74 & 5,62 & 8,70 & 6,73 \\
\hline C5 & 3,49 & 15,00 & 11,24 & 13,50 & 16,03 & 9,68 & 4,77 & 13,36 \\
\hline C6 & 1,93 & 1,71 & 1,64 & 1,71 & 2,10 & 1,82 & 2,22 & 1,85 \\
\hline $\mathrm{C} 7$ & 1,71 & 1,41 & 1,49 & 1,71 & 1,56 & 1,74 & 1,47 & 1,59 \\
\hline
\end{tabular}

E1 Actividad, E2 Empleo, E3 Desempleo, E4 Temporalidad, E5 Ocupaciones elementales, E6 Sobrecualificación, E7 Ganancia media.

B1 Hogares que llegan con dificultades a final de mes, B2 Gasto medio del hogar, B3 Personas por habitación, B4 Estado de salud percibida, B5 Estudios superiores.

R1 Hogares mixtos, R2 Convivencia de parejas en el hogar, R3 Hogares con menores dependientes, R4 Capacidad lingüística, R5 Aceptación.

C1 Regularidad documental, C2 Residencia de larga duración, C3 Nacionalización por residencia, C4 Sufragio activo, C5 Sufragio pasivo, C6 Escolaridad infantil, C7 Escolaridad postobligatoria.

Sombreados: rojo $=$ peor, verde $=$ mejor, blanco $=$ intermedio. Los dos puntos de corte que definen estos tres segmentos se establecen de forma que la tasa de variación entre el mínimo y el primer punto de corte sea igual que la tasa de variación entre el primer punto de corte y el segundo, e igual que la tasa de variación entre el segundo punto de corte y el máximo.

Fuente: elaboración propia a partir de diversas fuentes estadísticas.

2. Nordeste y, en menor medida, Noroeste, integran el grupo de regiones en las que las diferencias entre extranjeros y nacionales son especialmente amplias, a pesar de un contexto general menos adverso.

3. Madrid y Este son regiones de diferencias relativamente limitadas entre extranjeros y nacionales, muchas veces parecidas a la media nacional, en un contexto laboral menos adverso. 
4. En la región Centro es donde se observan diferencias pronunciadas entre extranjeros y nacionales en un contexto relativamente adverso.

Como se puede apreciar, estar en una posición más favorable, con menores tasas de paro en la región, no garantiza una mayor igualdad entre extranjeros y nacionales. De hecho, tiende a ocurrir lo contrario: las regiones más desfavorecidas se caracterizan por un mayor grado de igualdad. Aun así, esto no implica que una persona inmigrante en una región con mayor desigualdad esté en una situación peor que otra en una región más igualitaria. Nos encontramos, así, ante la ya citada integración a la baja, según la cual, en algunas comunidades autónomas, las diferencias son menores, pero a costa de una mayor precariedad social y económica de ambos colectivos.

Por su parte, los indicadores sobre las condiciones materiales (Bienestar) evidencian, a escala estatal, que la población inmigrante se encuentra en una situación desfavorecida en comparación con la autóctona. Concretamente, es el caso de las mediciones relativas a Hogares que llegan con dificultades a fin de mes y al Gasto anual medio de los hogares. En cambio, la distancia entre ambas poblaciones es menor en los cocientes relativos a Personas por habitación en el hogar o a Estudios superiores, y no observamos diferencias significativas en el caso de Salud percibida. Ahora bien, estas repercusiones tampoco son homogéneas desde una perspectiva territorial, de modo que la crisis estaría acentuando las brechas que se vislumbraban con anterioridad entre distintos territorios, sobre todo en los indicadores asociados a la situación económica.

En las marcadas diferencias interregionales, se aprecia un notable impacto de la composición de la población inmigrada residente en unas y otras partes del territorio. Las diferencias regionales en la estructura socioeconómica y laboral también contribuirían a explicar los datos más dispares. Ello es así sobre todo con respecto a la faceta más económica y material del bienestar, que se aborda por los tres primeros indicadores de este ámbito de medición. El indicador de Estudios superiores también aparece muy ligado a la especialización productiva de cada región, que es un factor que incide en la orientación de los flujos. Por su parte, el indicador que más se aleja de estas pautas es el del Estado de salud percibido; se observa una situación de equiparación entre alóctonos y autóctonos, siempre y cuando se comparen grupos de edad equivalentes (16-45 años).

Teniendo en cuenta los datos de la tabla 1, puede plantearse una tipología territorial, tomando como criterios los indicadores incluidos en los ejes de Empleo y Bienestar individual y en la que puede observarse que dichos indicadores tienden a empeorar conforme avanzamos en sentido meridional a lo largo del país.

1. Noroeste y Nordeste, donde los indicadores anteriormente citados muestran una magnitud manifiestamente más alta para la población nacional, y una sensiblemente más baja para la extranjera. 
2. Madrid, Centro y Este, que son regiones en las que los indicadores asociados a Empleo y Bienestar individual son intermedios o intermedio-bajos y las circunstancias de la población extranjera tienden a equipararse, aunque siempre por debajo de la población española.

3. Sur y Canarias, donde las magnitudes de los indicadores son menores a la media nacional y la diferencia entre los colectivos español y extranjero son menores, e incluso en algunos indicadores es mejor el nivel observado para la población extranjera.

En tercer lugar, si nos referimos a la media de España en el ámbito de las Relaciones sociales, para el subámbito de redes sociales, la situación muestra un cociente de Convivencia en el hogar - domicilios en los que viven las dos personas que componen la pareja - igualitario para parejas españolas y extranjeras, así como un cociente de Menores dependientes notablemente más positivo en hogares extranjeros. Aquí la edad de los inmigrantes desempeña un papel importante, al concentrarse en los segmentos de entre 25 y 44 años, propicios a la formación de parejas y familias.

Por lo que respecta al eje de las relaciones sociales, también se puede hablar de un perfil propicio a la integración, especialmente con respecto al indicador de Aceptación, ya que arroja un valor muy alto de actitudes positivas (o, cuanto menos, neutras) de la población española hacia la inmigrada. Sin embargo, los resultados obtenidos en Capacidad lingüística y Hogares mixtos no son tan favorables. Por ejemplo, es preocupante que solo la mitad de los extranjeros dominen uno o más de los idiomas oficiales.

A nivel de territorios NUTS-1, la situación en general es similar a la estatal: es favorable en lo que concierne a la calidad de las redes sociales, mientras que, en cuanto a las relaciones intergrupales, arroja datos menos positivos. De ello, se podría desprender que el nivel de integración es alto en cuanto a la disponibilidad de redes sociales por parte de las personas inmigrantes, pero no tanto en relación con los contactos entre españoles y extranjeros, aunque estos cuenten, mayoritariamente, con la aceptación de los primeros.

El patrón que se detecta en materia de relaciones sociales es bastante heterogéneo, aunque permite diferenciar tres tipos de regiones:

1. Nordeste, Madrid y Este, donde las diferencias entre extranjeros y nacionales son menos amplias o, como mucho, tan amplias como la media estatal, excepto para el indicador de hogares mixtos.

2. Las regiones Sur, Centro y Noroeste, con cocientes más desfavorables que la media estatal en por lo menos uno de los indicadores de cada subdimensión temática (calidad y configuración de las redes sociales, respectivamente).

3. Canarias, donde las diferencias entre extranjeros y nacionales son parecidas o menores que la media estatal, excepto por un indicador relativo a la calidad de las redes sociales, que denota esencialmente el dinamismo demográfico de unos y otros (presencia de menores dependientes en el hogar). 
Por último, en los diversos subámbitos de la Ciudadanía (civil, política y social), la situación es divergente a escala nacional. Pese al contexto de crisis, los datos trazan un perfil caracterizado por un buen nivel de acceso a la ciudadanía cívica: una proporción muy elevada de extranjeros se encontraría en situación administrativa regular, y esto es una situación propicia para los procesos de integración. Asimismo, los datos son positivos con respecto a la obtención de la residencia de larga duración. En cuanto al acceso a la nacionalidad por residencia, el valor se sitúa en un nivel similar al de países europeos de mayor tradición migratoria. Por otra parte, los indicadores relacionados con la participación política se encuentran en valores propios de países con migraciones recientes, aunque no por ello satisfactorios (Spencer, 2006). Tanto los cocientes del indicador de Personas inscritas para votar como el de Personas electas (solo aplicable a las nacidas en el extranjero con nacionalidad de un país de la UE) arrojan valores mínimos y evidencian notables problemas en este subámbito. Con respecto a la ciudadanía social, los resultados muestran que hay una brecha entre el alumnado extranjero y el español, tanto en la etapa preobligatoria como en la postobligatoria.

A escala regional, todos los indicadores obtienen puntuaciones superiores a 1 en todas las regiones. Ahora bien, estas son especialmente elevadas en el caso de la Obtención de la nacionalidad y en el de Sufragio pasivo. Se observa que, en Regularidad documental y en Residencia de larga duración, casi todas las regiones presentan valores positivos en comparación con la media nacional, con la excepción de Madrid para el primero y de Noroeste para el segundo. Por su parte, los indicadores sobre escolarización en etapas no obligatorias sugieren que el ejercicio real de los derechos sociales es determinado por circunstancias específicas. En este sentido, interpretaríamos el hallazgo de que, para la mayoría de las regiones, un valor relativamente favorable en una etapa educativa vaya ligado a una posición desfavorable o, como mucho, intermedia con respecto a otro tramo o a otra etapa educativa.

En definitiva, dada la acusada heterogeneidad que presenta la panorámica en el ámbito de la Ciudadanía, lo cierto es que no se detectan patrones claros que delimiten diversos tipos regionales. En donde se detectan más dificultades son en el Noroeste y en Canarias. Ambas arrojan cocientes relativamente altos en casi todas las mediciones. En un nivel intermedio en cuanto a los datos obtenidos, se sitúan Sur, Este y Centro. El Nordeste y Madrid son las regiones que presentan los resultados más propicios para la integración.

\subsection{Indices sintéticos y clústers}

La agrupación de las regiones NUTS-1 en función de los resultados del análisis clúster resulta en tres grupos en cada uno de los cuatro ámbitos. En la tabla 2, se exponen estas agrupaciones, indicando también los índices sintéticos de las regiones según cada ámbito temático. Se observa una clara relación entre la pertenencia a un grupo del clúster y el nivel del índice sintético. Solo un limitado número de casos se salen de esta regularidad. A continuación, se realiza 
Tabla 2. Agrupación de regiones NUTS-1 en función del análisis clúster jerárquico tipo Ward (cuatro ámbitos)

\begin{tabular}{|c|c|c|c|c|c|c|c|c|}
\hline \multirow[b]{2}{*}{ Ámbitos } & \multirow[b]{2}{*}{ Grupos } & \multicolumn{7}{|c|}{ Regiones NUTS-1 } \\
\hline & & Noroeste & Nordeste & Madrid & Centro & Este & Sur & Canarias \\
\hline \multirow[t]{3}{*}{ Empleo } & 1 & 1,60 & 1,73 & & & 1,66 & & \\
\hline & 2 & & & 1,58 & 1,50 & & 1,53 & \\
\hline & 3 & & & & & & & 1,30 \\
\hline \multirow[t]{3}{*}{ Bienestar } & 1 & & 1,57 & & & & & \\
\hline & 2 & 1,38 & & 1,32 & 1,27 & 1,23 & 1,17 & \\
\hline & 3 & & & & & & & 1,14 \\
\hline \multirow[t]{3}{*}{ Relaciones } & 1 & & & & 1,60 & 1,58 & 1,66 & \\
\hline & 2 & & 1,43 & 1,39 & & & & 1,40 \\
\hline & 3 & 1,42 & & & & & & \\
\hline \multirow[t]{3}{*}{ Ciudadanía } & 1 & & & & 4,05 & & 3,98 & \\
\hline & 2 & & 3,99 & 3,58 & & 4,25 & & \\
\hline & 3 & 3,29 & & & & & & 3,55 \\
\hline
\end{tabular}

Fuente: elaboración propia a partir de diversas fuentes estadísticas.

Nota: incluidos en las celdas los valores de los índices sintéticos.

una descripción de los resultados por ámbitos temáticos, en los que se incluyen referencias explícitas a los casos excepcionales.

En el ámbito de Empleo, las diferencias entre los índices sintéticos computados se ubican al Nordeste, al Noroeste y al Este en posiciones de mayor desigualdad entre extranjeros y nacionales. En el otro extremo se encuentra Canarias, cuyo índice sintético es el más bajo, seguido por el Centro y el Sur. A su vez, también la agrupación a través del clúster ubica a Canarias en un extremo, y al Nordeste, Noroeste y Este, en el otro. Las otras tres regiones restantes forman un grupo intermedio de cierta heterogeneidad interior, según indican sus índices sintéticos. Madrid muestra mayores desigualdades, mientras que en el Centro y en el Sur estas son menores. En cuanto a la heterogeneidad interna, esta se hace especialmente visible en el Este, y solo en menor medida en el Noroeste, en el Nordeste y en el Centro.

En el ámbito temático de Bienestar, el Nordeste destaca por un mayor valor de su índice sintético. En el polo opuesto, se sitúa de nuevo Canarias; mientras que el resto de regiones se ubica en posiciones intermedias. El análisis clúster confirma las posiciones extremas (Nordeste, Canarias) como grupos a título propio, así como la constitución de una tercera agrupación por todas las regiones restantes. En este amplio grupo, los índices sintéticos de Madrid y Noroeste son mayores que los de Centro, Este y Sur. En el indicador B1, las regiones Centro y Sur se parecen por sus bajos cocientes, ejemplos de menores niveles de desigualdad frente a la población nacional, en comparación con las puntuaciones mucho más elevadas de Noroeste, Madrid y Este. En resumidas cuentas, el grupo intermedio comprende realidades bastante dispares, si nos referimos a indicadores concretos. 
El ámbito temático de Relaciones sociales muestra desigualdades claramente más pronunciadas en las regiones Sur, Centro y Este, a tenor de los índices sintéticos. Nuevamente, el análisis clúster confirma esta agrupación. Llama la atención que, en estas mismas regiones, se habían observado niveles relativamente bajos de desigualdad en Empleo y Bienestar. Las cuatro regiones restantes tienen índices sintéticos más bajos y relativamente similares entre sí, pero el análisis clúster las separa en dos grupos; por un lado, el Noroeste y, por otro, Nordeste, Madrid y Canarias. Noroeste se separa de las últimas, a pesar de un índice sintético de valor medio dentro de este grupo, debido a su posición extrema en los indicadores R1 (cociente bajo en Hogares mixtos) y R5 (cociente alto en Aceptación). En estos indicadores, Canarias obtiene un perfil contrapuesto al Noroeste (alto en R1; bajo en R5). En consecuencia, aunque estas regiones tengan índices sintéticos similares, los perfiles que dan lugar a dichos valores son claramente diferentes.

Otra vez más, la heterogeneidad interna de las regiones NUTS-1 es amplia en el Este. Se observan claras diferencias entre Cataluña y las otras comunidades autónomas pertenecientes a esta agrupación. Al igual que en el ámbito del Bienestar, en las otras regiones NUTS-1 la carencia de datos sobre las comunidades autónomas impide una comparación sistemática.

El ámbito temático de Ciudadanía cuenta con agrupaciones similares al ámbito de Relaciones sociales. Las regiones Este, Centro, Nordeste y Sur presentan mayores valores en los índices sintéticos y, de nuevo, el análisis clúster confirma esta agrupación con la salvedad de las regiones Este y Nordeste, integradas en el grupo intermedio junto con Madrid. Noroeste y Canarias, con los valores más bajos en los índices sintéticos, forman el tercer grupo que coincide con el análisis clúster. Estas incongruencias entre la agrupación jerárquica del clúster y los índices sintéticos están relacionadas, entre otros aspectos, con las amplias diferencias en el indicador C5 (Personas inscritas para votar en elecciones locales), el peso que retiene este indicador por su amplia escala en el indicador sintético y el valor extremo que adquiere en la región Este. Aparte del valor elevado en el indicador C5, el perfil de la región Este es más bien de valores medios o bajos en los demás indicadores.

La heterogeneidad interna a nivel NUTS-1 se refleja en los datos de comunidades autónomas, siempre que estén disponibles. En la región Este, los indicadores C1 (Regularidad documental), C6 (Escolaridad infantil) y C7 (Escolaridad postobligatoria) muestran una homogeneidad relativa, y es en los indicadores C3 (Naturalización) y C5 (Personas inscritas para votar) donde se observan las mayores diferencias. En las regiones restantes, las diferencias son menores y se manifiestan con claridad solo en el indicador C5.

Por último, también computamos una clasificación genérica a través de los índices sintéticos generales, basados en la combinación ponderada de los cuatro índices sintéticos por ámbito, junto a los grupos creados por el análisis clúster (gráfico 1 y tabla 3).

La agrupación jerárquica genera tres grupos: Noroeste, Nordeste y Madrid; Centro, Este y Sur, y Canarias. Esta última región ofrece el índice sintético 
Gráfico 1. Mapa de la clasificación jerárquica de las regiones NUTS-1 en el conjunto de ámbitos, según el Índice sintético general de integración

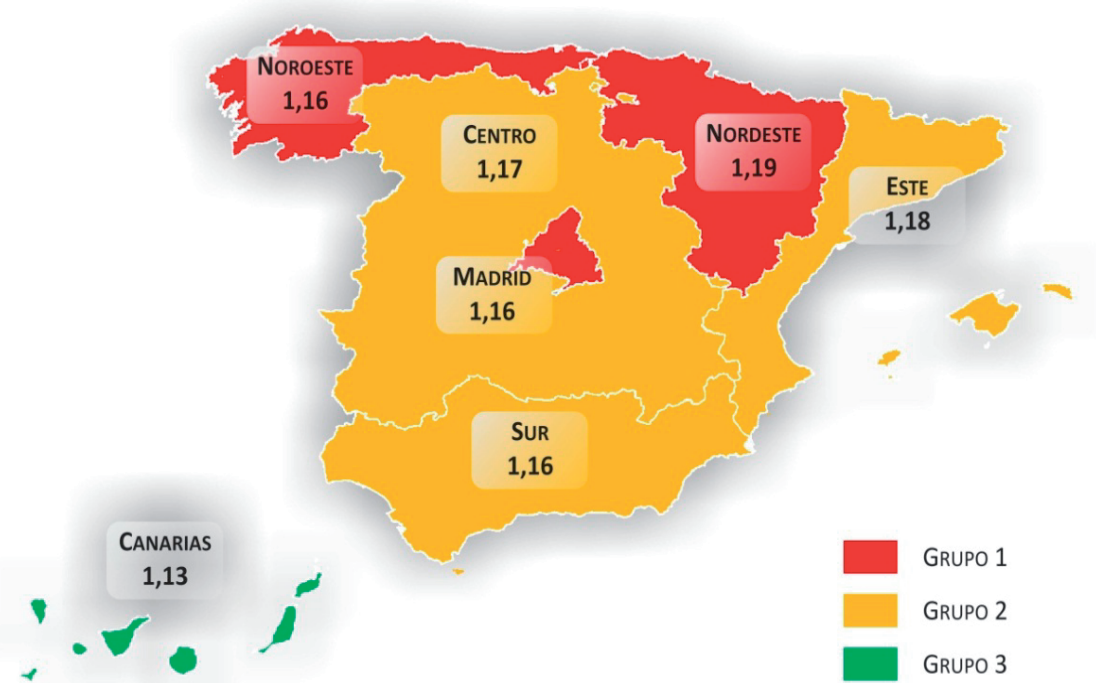

Fuente: elaboración propia a partir de diversas fuentes estadísticas.

Tabla 3. Agrupación de las regiones NUTS-1 en función del análisis clúster jerárquico tipo Ward (índices sintéticos generales de integración)

\begin{tabular}{cccccccc}
\hline Grupo & Noroeste & Nordeste & Madrid & Centro & Este & Sur & Canarias \\
\hline 1 & 1,16 & 1,19 & 1,16 & & & & \\
2 & & & & 1,17 & 1,18 & 1,16 & \\
3 & & & & & & & 1,13 \\
\hline
\end{tabular}

Fuente: elaboración propia a partir de diversas fuentes estadísticas.

Nota: incluidos en las celdas los índices sintéticos generales.

general más bajo y queda claramente separada también en el clúster. Los otros dos grupos muestran índices sintéticos heterogéneos dentro de cada grupo; por ejemplo: Nordeste y Este tienen altos índices sintéticos, pero están en grupos (clústers) diferentes. La causa de esta divergencia estriba en los perfiles opuestos de ambas regiones en los ámbitos Empleo y Bienestar versus Relaciones sociales y Ciudadanía. Los efectos de compensación entre ámbitos, pese a referirse a perfiles diferentes, reducen las diferencias en el índice sintético general, esto es, se llega a índices sintéticos similares a través de caminos distintos. En resumidas cuentas, y de forma parecida a lo detectado en los análisis de cada ámbito, la agrupación mediante clústers mantiene la especificidad de los perfiles subyacentes en mayor medida que la cuantificación de las desigualdades globales mediante índices sintéticos generales. 


\section{Conclusiones}

Aprovechando un amplio abanico de fuentes, el sistema de medición presentado aquí permite comparar los perfiles de integración en cuatro ámbitos de la realidad social a escala regional. Como primer hallazgo destacable, cabe señalar que, en prácticamente todas las vertientes de dichos procesos, las diferencias entre regiones constituyen matices más o menos importantes con respecto a una pauta común a escala estatal. Es decir, como regla general, se observa que los procesos de integración están evolucionando mejor en algunos ámbitos, como, por ejemplo, en el de las Relaciones sociales, que en otros. No sorprende que el Empleo resalte por una situación desfavorable en todo el territorio nacional.

Dicho esto, mediante varias técnicas estadísticas (cocientes, índices sintéticos y análisis clúster), pudimos discernir tres perfiles regionales. En el Nordeste y en el Noroeste de España, así como en la Comunidad de Madrid, los datos apuntan a unos mayores niveles de desigualdad entre las personas inmigrantes y autóctonas en cuanto a aspectos materiales (Empleo y Bienestar); sin embargo, en dichas regiones, la desigualdad con respecto a derechos y convivencia (Relaciones sociales y Ciudadanía) es menor, siempre en comparación con la media nacional. En cambio, en el Centro, en el Este y en el Sur del país, se da la situación opuesta: menor desigualdad material combinada con mayor desigualdad en derechos y convivencia. Un tercer perfil lo representa Canarias, que emerge como la única región con desigualdades menores entre las poblaciones extranjera y nacional en los cuatro ámbitos de medición.

Estos perfiles diferenciales son atribuibles a un compendio de factores relacionados con el territorio, entre los que destacan la estructura y la especialización económica, los niveles de bienestar material, el grado de desarrollo y cobertura de servicios básicos, así como los atributos de la población inmigrante. Todo ello condiciona tanto la composición de la población inmigrada y su situación como el nivel alcanzado por la población autóctona con respecto de los parámetros establecidos. Este nivel, según nuestra aproximación operativa al concepto de integración, marca el listón para la equiparación progresiva o el distanciamiento entre ambas poblaciones.

¿Cuáles son los factores que explican estos perfiles contrapuestos? Primero, las regiones con mayor desigualdad entre extranjeros y nacionales en los ámbitos de Empleo y Bienestar cuentan con segmentos amplios de nacionales de ingresos medios y altos. Aquí, la posición de las personas inmigrantes es desfavorable en comparación con las españolas, pero no necesariamente en comparación con los extranjeros en otras regiones. Segundo, la menor desigualdad con respecto a las Relaciones sociales y la Ciudadanía puede ser el resultado de una mayor dotación de recursos públicos y de la composición de la inmigración. Tercero, la menor desigualdad económica en las regiones Centro, Este y Sur tiene relación con el peso de aquella parte del territorio que combina amplias capas de nacionales de ingresos bajos con extranjeros que desempeñan funciones laborales relativamente similares. Este argumento también es aplicable a Canarias. Cuarto, la mayor desigualdad en Ciudadanía 
y Relaciones sociales puede estar vinculada a un menor grado de desarrollo del Estado de bienestar, debido a la menor disponibilidad de recursos públicos y a la composición de la inmigración, con una elevada participación de personas inmigrantes comunitarias, por un lado, y de recién llegados de terceros países y en situación laboral precaria, por otro. Ahora bien, cabe resaltar también que estos perfiles particulares desembocan en índices sintéticos generales similares, al compensarse las desviaciones en una y otra dirección. Por lo tanto, resulta analíticamente más enriquecedor contemplar los índices sintéticos específicos para cada ámbito temático.

Obviamente, es inevitable que haya limitaciones a la hora de construir un sistema de medición, de modo que es preciso mencionarlas para no difuminar sus virtudes. Somos conscientes de que aún hay lagunas en áreas con dificultades para una medición más exhaustiva, como Relaciones sociales o Ciudadanía. Asimismo, a menudo, las fuentes carecen de información sobre el país de nacimiento y de cobertura estadística para desglosar entre distintas nacionalidades. Por ello, nuestras mediciones se refieren a la dicotomía existente entre nacionalidad extranjera versus nacionalidad española. A su vez, la desagregación a escala autonómica no fue posible debido a la poca representatividad de las muestras de algunas encuestas. Para superar este escollo, se utilizan las agrupaciones territoriales NUTS-1 de Eurostat. Por último, el sistema de indicadores se centra en las diferencias entre extranjeros y nacionales en cada región, y no refleja las divergencias en las condiciones de vida de las personas inmigrantes. En este sentido, el resultado no contesta a la pregunta de cuál es la mejor región para vivir siendo inmigrante, ya que la medición comparada entre inmigrantes y nativos del grado de integración puede ofrecer deducciones inicuas. De hecho, surge una cuestión de gran interés: ¿qué es mejor para una persona extranjera, estar en una mala situación, pero sin diferencias con la población nativa, o estar mejor que las personas extranjeras que residen en otra región, pero con mayores diferencias con la población nativa? Ello deja abierto el debate sobre la medición de este concepto y, por lo tanto, la propia mejora del sistema propuesto.

Como contrapunto a estas limitaciones, este novedoso sistema de medición de la integración de las personas inmigrantes en las regiones españolas realiza por lo menos tres aportaciones.

En primer lugar, la notoria multidimensionalidad del concepto de integración es captada mejor que por otras propuestas de medición, incluyendo la Declaración de Zaragoza, al abarcar también aspectos que suelen eludir la medición estadística, como, por ejemplo: la calidad y la composición de las redes sociales. Sin ser perfecto, el sistema propuesto aquí extiende la medición a procesos que nunca habían sido objeto de medición rigurosa, pese a su reconocida importancia para la integración.

En segundo lugar, el sistema sienta las bases para un seguimiento longitudinal de estos procesos. La actualidad sigue estando marcada por los efectos de la crisis y, por ello, un sistema de medición resulta especialmente necesario en estos momentos. Todos los indicadores elegidos ofrecen la posibilidad de 
realizar actualizaciones periódicas. Además, al basarse en datos provenientes de estadísticas o registros oficiales, no se necesita generar información primaria, con los correspondientes gastos añadidos.

$Y$, en tercer lugar, este sistema de medición es el primero en abordar los procesos de integración de las personas inmigrantes a escala no ya nacional, sino regional. La realidad social varía en las diversas regiones españolas. Esta constatación es pertinente, puesto que, en temas de extranjería e inmigración, el predominio de la legislación nacional podría inducir a subestimar la relevancia de la dimensión regional en los procesos de integración, en los que, además, su medición estadística se solía dar por imposible a efectos prácticos. En este sentido, parece obvio que el uso de indicadores debe complementarse con un análisis de contexto que incluya la situación socioeconómica, el tipo de inmigración u otro modelo de condicionantes en este proceso.

\section{Referencias bibliográficas}

Aguado, M.a Teresa (1997). Educación multicultural: Su teoría y su práctica. Madrid: Universidad Nacional de Educación a Distancia.

Alba, Richard y Nee, Victor (1997). «Rethinking Assimilation Theory for a New Era of Immigration». International Migration Review [en línea], 4 (31), 826-874. $<$ http://dx.doi.org/10.2307/2547416>.

Arango, Joaquín (1999). "Becoming a Country of Immigration at the End of the XXth Century: The Case of Spain». En: King, Russel; Lazaridis, Gabriella y Tsardanidis, Chalambaros (eds.). Eldorado or Fortress?: Migration in Southern Europe. Londres: Macmillan Press, 253-276.

Blanco, Cristina (2000). Las migraciones contemporáneas. Madrid: Alianza Editorial.

Bosswick, Wolfgang y Heckmann, Friedrich (2006). Integration of migrants: Contribution of local and regional authorities [en línea]. Dublín: European Foundation for the Improvement of Living and Working Conditions. $<$ http://bit.ly/1FrwCcW> [Consulta: 23 marzo 2013].

BraH, Avtar (1996). Cartographies of Diaspora: Contesting identities. Londres: Routledge.

CACHón, Lorenzo (2008). «La integración de y con los migrantes en España: Debates teóricos, políticas y diversidad territorial». Política y Sociedad, 45 (1), 205-235.

Colectivo Ioé (2008). Barómetro social de España: Análisis del periodo 1994-2006. Madrid: Traficantes de Sueños.

Comité de las Regiones (2012). «Dictamen del Comité de las Regiones: Nueva Agenda Europea para la Integración». Diario Oficial de la Unión Europea [en línea], n. ${ }^{\circ}$ C 113, 11-16 (18 abril 2012). <http://www.bizkaia.net/ogasuna/europa/pdf/ documentos/12-C113-04.pdf> [Consulta: 9 noviembre 2013].

Conferencia Ministerial Europea sobre Integración (2010). Declaración de Zaragoza. Zaragoza: Presidencia española de la Unión Europea, 15 y 16 de abril de 2010.

Dassetto, Felice (1990). «Pour une théorie des cycles migratoires». En: Bastenier, Albert y DassetTo, Felice (eds.). Immigrations et nouveaux pluralismes: Une confrontation des sociétés. París: De Boeck Université, 11-39.

ENGELEN, Eward (2003). "Conceptualizing Economic Incorporation: From "Institutional Linkages" to "Institutional Hybrids"». Ensayo presentado en The 
Conference on Conceptual and Methodological Developments in the Study of International Migration. Princeton University, 23-25 de mayo.

Entzinger, Han y Biezeveld, Renske (2003). Benchmarking in Immigrant Integration [en línea]. Rotterdam: European Research Centre on Migration and Ethnic Relation. <http://bit.ly/1c7DzTX> [Consulta: 7 abril 2013].

Eurostat (2011). Indicators of immigrant integration: A pilot study [en línea]. Luxemburgo: Publications Office of the European Union. <http://bit.ly/1CC4zAe> [Consulta: 29 agosto 2013].

FREEMAN, Gary (2004). "Immigrant incorporation in Western Democracies». International Migration Review [en línea], 38, 945-969. <http://dx.doi.org/10.1111/j.1747-7379.2004.tb00225.x>.

García Cívico, Jesús (2011). «Sobre el proceso de integración social del inmigrante en España: La cuestión de los indicadores». Revista Electrónica del Instituto de Investigaciones "Ambrosio L. Gioja» [en línea], año V (7), 50-77. <http://bit.ly/1sEQsuN> [Consulta: 18 diciembre 2012].

Giménez, Carlos (1996). «La integración de los inmigrantes y la interculturalidad». Arbor, 607, 119-149.

Godenau, Dirk; Rinken, Sebastian; Martínez de Lizarrondo, Antidio y Moreno, Gorka (coords.) (2014). La integración de los inmigrantes en las regiones españolas: Una propuesta de medición. Madrid: Observatorio Permanente de la Inmigración. Ministerio de Empleo y Seguridad Social. Documentos, 30.

Gordon, Milton (1964). Assimilation in American Life. Nueva York: Oxford University Press.

- (1975). «Toward a General Theory of Racial and Ethnic Group Relations». En: Glazer, Nathan y Moynihan, Daniel (eds.). Ethnicity: Theory and Experience. Cambridge: Harvard University Press, 84-110.

Guiraudon, Virginie (2000). "The Marshallian Tryptich Reordered: The Role of Courts and Bureaucracies in Furthering Migrants' Social Rights». En: Bommes, Michael y Geddes, Andrew (eds.). Immigration and Welfare: Challenging the Borders of the Welfare State. Londres: Routledge, 72-89.

Hammar, T. (1990). Democracy and the Nation State: Aliens, Denizens and Citizens in a World of International Migration. Warwick: Avebury.

Hammar, Tomas y Lithman, Yngve (1989). «La integración de los migrantes: Experiencias, conceptos y políticas». En: OCDE. El futuro de las migraciones. Madrid: Ministerio de Trabajo y Seguridad Social, 347-385.

Heckmann, Friedrich y Schnapper, Dominique (2003). The integration of immigrants in European societies. Stuttgart: Lucius and Lucius.

Huddleston, Thomas; Niessen, Jan y Dag Tuaden, Jasper (2013). Using EU indicators of immigrant integration: Final report for Directorate-General for Home Affairs [en línea]. Bruselas: European Commission. <http://bit.ly/1TMSBxo> [Consulta: 27 agosto 2013].

Huddleston, Thomas; Niessen, Jan; Ni Chaoimh, Eadaoin y White, Emilie (2011). Migrant Integration Policy Index 2011. MIPEX III. Bruselas: British Council. Migrant Policy Group.

IzQuierdo, Antonio y León-Alfonso, Sandra (2008). «La inmigración hacia dentro: Argumentos sobre la necesidad de coordinación de las políticas de inmigración en un Estado multinivel». Politica y Sociedad, 1 (45), 11-39.

Joppke, Christian (1999). Immigration and the Nation State. Oxford: Oxford University Press. 
Joppke, Christian y Morawska, Ewa (2003). "Integrating immigrants in liberal nation-states: Policies and practices». En: Joppke, Christian y Morawska, Ewa (eds.). Toward Assimilation and Citizenship: Immigration in Liberal Nation-States. Basingstoke: Palgrave Macmillan, 1-36.

Lahav, Gallya (1998). «Immigration and the State: The Devolution and Privatisation of Immigration Control in the EU». Journal of Ethnic and Migration Studies [en línea], 24 (4), 675-694. <http://dx.doi.org/10.1080/1369183x.1998.9976660>.

LAMBERT, Wallace y TAYLOR, Donald (1988). «Assimilation versus Multiculturalism: The Views of Urban Americans». Sociological Forum [en línea], 3 (1), 72-88. <http://www.jstor.org/stable/684622>.

Lamo de Espinosa, Emilio (1995). «Fronteras culturales». En: LAmo de Espinosa, Emilio (ed.). Culturas, Estados, Ciudadanos: Una aproximación al multiculturalismo en Europa. Madrid: Alianza / Fundación Ortega y Gasset, 13-79.

Laparra, Miguel y PÉrez, Begoña (coord.) (2008). «La exclusión social en España: Un espacio diverso y disperso en intensa transformación». En: Fundación FOESSA. VI Informe sobre la exclusión y desarrollo social en España. Conclusiones. Madrid: Fundación FOESSA, 53-88.

Martínez de Lizarrondo, Antidio (2009). «La integración de inmigrantes en España: El modelo patchwork». Migraciones, 26, 115-146.

Ministerio de Trabajo y Asuntos Sociales (2007). Indicadores de Integración de Inmigrantes: Propuesta para contribuir a la elaboración de un sistema de indicadores comunes de la integración. Madrid.

OECD (2012). Settling In: OECD Indicators of immigrant integration 2012 [en línea]. París: OECD Publishing. <http://www.oxydiane.net/IMG/pdf/OECD_Immi grants_2012.pdf> [Consulta: 27 febrero 2013].

Penninx, Rinus; Kraal, Karen; Martinello, Marco y Vertovec, Steven (2004). Citizenship in European Cities: Immigrants, local politics and integration policies. Aldershot: Ashgate.

Phalet, Karen y Swyngedouw, Marc (2003). «Measuring migrant integration: The case of Belgium». Studi Emigrazione/Migration Studies [en línea], XL (152), 773803. <http://adapt.it/adapt-indice-a-z/wp-content/uploads/2014/04/phalet_swyn gedouw.pdf> [Consulta: 2 marzo 2012].

PorTes, Alejandro y Borocz, Jozsef (1989). «Contemporary Immigration: Theoretical Perspectives on its Determinants and Modes of Incorporation». International Migration Review [en línea], 23, 606-630. <http://dx.doi.org/10.2307/2546431>.

Provansal, Danielle (1999). “¿De qué migración hablamos?: Desde los conceptos a las prácticas sociales». En: Checa, Francisco y Soriano, Encarna (eds.). Inmigrantes entre nosotros: Trabajo, cultura y educación intercultural. Barcelona: Icaria, 17-31.

Richmond, Anthony (1988). "Socio-cultural adaptation and conflict in immigrant-receiving countries». En: STAHL, Charles (ed.). International migration today: Emerging issues [en línea]. Unesco-University of Western Australia: Centre for Migration and Development Studies, 109-124. <http://unesdoc.unesco.org/ images/0005/000579/057956eb.pdf> [Consulta: 2 febrero 2013].

Rodríguez IzQuierdo-SERrano, Miriam (2013). «Inmigración, integración y competencias en materia de empleo: El papel, y el reto, de las Comunidades Autónomas». Lex Social, 3 (1), 34-40. 
RodríGuez Rojo, Martín (2006). «Interculturalismo, ciudadanía cosmopolita y educación intercultural». Revista Interuniversitaria de Formación del Profesorado, 55, 17-38.

Schierup, Carl-Ulrik y Ålund, Aleksandra (1986). Will They Still Be Dancing?: Integration and Ethnic Transformation among Yugoslav Immigrants in Scandinavia. Estocolmo: Almqvist y Wiksell International.

Soysal, Yasemin (1994). Limits of Citizenship: Migrants and Postnational Membership in Europe. Chicago: Chicago University Press.

SPENCER, Sarah (2006). Social Integration of Migrants in Europe: A Review of the European Literature 2000-2006 [en línea]. Oxford: Centre on Migration, Policy and Society. <http://bit.ly/1Uj9QpR> [Consulta: 3 septiembre 2012].

Telles, Edward y Ortiz, Vilma (2011). Generaciones excluidas: Mexicano-estadounidenses, asimilación y raza. Madrid: Centro de Investigaciones Sociológicas.

Thomas, Wiliam y Znaniecki, Florian (1996). The Polish Peasant in Europe and America. Chicago: University of Illinois.

Vertovec, Steven (2010). «Towards post-multiculturalism?: Changing communities, conditions and contexts of diversity». International Social Science Journal [en línea], 61, 83-95.

$<$ http://dx.doi.org/10.1111/j.1468-2451.2010.01749.x>. 


\section{Anexo}

Tabla 4. Fórmulas de cómputo y fuentes estadísticas de origen de los indicadores

\begin{tabular}{|c|c|c|c|}
\hline Dimensión & Indicador & $\begin{array}{c}\text { Fórmula del indicador (fase inicial, no incluye } \\
\text { la conversión en cociente de valores para } \\
\text { extranjeros y nacionales) }\end{array}$ & Fuente \\
\hline \multirow[t]{7}{*}{ Empleo } & Actividad & $\begin{array}{l}\text { Tasa de actividad }=\left(N^{0}{ }^{0} \text { total de activos / población }\right. \\
\text { potencialmente activa [16 y más años] })^{*} 100 .\end{array}$ & Encuesta de Población Activa (EPA). \\
\hline & Empleo & $\begin{array}{l}\text { Tasa de empleo }=\left(N^{0}{ }^{0} \text { total de ocupados / población }\right. \\
16-64 \text { años })^{*} 100 .\end{array}$ & Encuesta de Población Activa (EPA). \\
\hline & Paro & $\begin{array}{l}\text { Tasa de paro }=\left(\mathrm{N}^{\circ}{ }^{0} \text { total de parados } / \mathrm{N}^{\circ}{ }^{0} \text { total de }\right. \\
\text { activos })^{*} 100 .\end{array}$ & Encuesta de Población Activa (EPA). \\
\hline & Temporalidad & $\begin{array}{l}\text { Tasa de temporalidad }=\left(\mathrm{N} .^{\circ} \text { de ocupados por cuenta }\right. \\
\text { ajena con contrato temporal } / \mathrm{N} .{ }^{\circ} \text { total de ocupados } \\
\text { por cuenta ajena }{ }^{*} 100 .\end{array}$ & Encuesta de Población Activa (EPA). \\
\hline & $\begin{array}{l}\text { Ocupaciones } \\
\text { elementales }\end{array}$ & $\begin{array}{l}\text { Tasa de ocupaciones elementales = (población } \\
\text { ocupada en ocupaciones elementales / población } \\
\text { ocupada total) }{ }^{*} 100 \text {. }\end{array}$ & Encuesta de Población Activa (EPA). \\
\hline & Sobrecualificación & $\begin{array}{l}\text { Tasa de sobrecualificación }=\left(\mathrm{N}^{\circ}{ }^{\circ} \text { de personas con }\right. \\
\text { estudios secundarios o superiores en ocupaciones } \\
\text { elementales / total de ocupados con estudios secun- } \\
\text { darios y superiores) }{ }^{*} 100 .\end{array}$ & Encuesta de Población Activa (EPA). \\
\hline & Ganancia media & Ganancia media anual por trabajador. & Encuesta Anual de Estructura Salarial. \\
\hline \multirow[t]{5}{*}{ Bienestar } & $\begin{array}{l}\text { Hogares que llegan con } \\
\text { dificultad a fin de mes }\end{array}$ & $\begin{array}{l}\text { Número de hogares que llegan con dificultad a fin } \\
\text { de mes sobre el total de hogares. }\end{array}$ & $\begin{array}{l}\text { Encuesta de Condiciones de Vida } \\
\text { (ECV). }\end{array}$ \\
\hline & Gasto medio del hogar & $\begin{array}{l}\text { Gasto total en euros de los hogares, dividido por } \\
\text { el total de hogares. }\end{array}$ & $\begin{array}{l}\text { Encuesta de Presupuestos Familiares } \\
\text { (EPF). }\end{array}$ \\
\hline & Personas por habitación & $\begin{array}{l}\text { Número de personas que conviven en el hogar, dividi- } \\
\text { do por el número total de habitaciones de la vivienda. }\end{array}$ & $\begin{array}{l}\text { Encuesta de Condiciones de Vida } \\
\text { (ECV). }\end{array}$ \\
\hline & Estado de salud & $\begin{array}{l}\text { Población entre } 16 \text { y } 45 \text { años con estado de salud } \\
\text { percibida como buena o muy buena, dividido por el } \\
\text { total de población entre } 16 \text { y } 45 \text { años. }\end{array}$ & $\begin{array}{l}\text { Encuesta de Condiciones de Vida } \\
\text { (ECV). }\end{array}$ \\
\hline & Estudios superiores & $\begin{array}{l}\text { Población mayor de } 16 \text { años con estudios superiores } \\
\text { terminados, dividido por el total de población mayor de } \\
16 \text { años con estudios terminados. }\end{array}$ & Encuesta de Población Activa (EPA). \\
\hline \multirow[t]{5}{*}{$\begin{array}{l}\text { Relaciones } \\
\text { sociales }\end{array}$} & Hogares mixtos & $\begin{array}{l}\text { Proporción de hogares en los que conviven personas } \\
\text { con nacionalidad extranjera y española, sobre el total } \\
\text { de hogares en los que, por lo menos, una persona } \\
\text { tiene nacionalidad extranjera. }\end{array}$ & $\begin{array}{l}\text { Encuesta de Presupuestos Familiares } \\
\text { (EPF). }\end{array}$ \\
\hline & $\begin{array}{l}\text { Convivencia de parejas } \\
\text { en el hogar }\end{array}$ & $\begin{array}{l}\text { Proporción de hogares en los que el sustentador } \\
\text { principal vive en pareja, sobre el total de hogares. }\end{array}$ & $\begin{array}{l}\text { Encuesta de Presupuestos Familiares } \\
\text { (EPF). }\end{array}$ \\
\hline & $\begin{array}{l}\text { Hogares con menores } \\
\text { dependientes }\end{array}$ & $\begin{array}{l}\text { Proporción de hogares en los que viven menores } \\
\text { dependientes, sobre el total de hogares. }\end{array}$ & $\begin{array}{l}\text { Encuesta de Presupuestos Familiares } \\
\text { (EPF). }\end{array}$ \\
\hline & Capacidad lingüística & $\begin{array}{l}\text { Proporción sobre el conjunto de la población con } \\
\text { nacionalidad extranjera de las personas capaces de } \\
\text { comunicarse en, por lo menos, una de las lenguas } \\
\text { oficiales vigentes en España. }\end{array}$ & $\begin{array}{l}\text { Encuesta sobre Participación de la } \\
\text { Población Adulta en Actividades de } \\
\text { Aprendizaje. }\end{array}$ \\
\hline & Aceptación & $\begin{array}{l}\text { Proporción sobre el conjunto de la población } \\
\text { española de quienes consideran que la presencia } \\
\text { de inmigrantes contribuye a mejorar (o a no empeorar } \\
\text { significativamente) la calidad de vida. }\end{array}$ & Encuesta Social Europea. \\
\hline
\end{tabular}




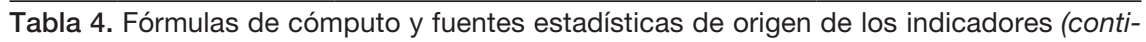
nuación)

\begin{tabular}{|c|c|c|c|}
\hline Dimensión & Indicador & $\begin{array}{c}\text { Fórmula del indicador (fase inicial, no incluye } \\
\text { la conversión en cociente de valores para } \\
\text { extranjeros y nacionales) }\end{array}$ & Fuente \\
\hline \multirow[t]{9}{*}{ Ciudadanía } & $\begin{array}{l}\text { Regularidad } \\
\text { documental }\end{array}$ & $\begin{array}{l}\text { Tasa de regularidad }=\left(\left[\mathrm{N} .{ }^{0} \text { de nacionales de }\right.\right. \\
\text { terceros países empadronados - Nacionales de } \\
\text { terceros países con autorización de residencia } \\
\text { en vigor + Nacionales de terceros países con } \\
\text { autorización de estancia por estudios] / Naciona- } \\
\text { les de terceros países empadronados })^{\star} 100 \text {. }\end{array}$ & Padrón de Habitantes. \\
\hline & & & $\begin{array}{l}\text { Registro de Extranjeros con } \\
\text { Certificado de Registro o Tarjeta } \\
\text { de Residencia en Vigor }\end{array}$ \\
\hline & & & $\begin{array}{l}\text { Registro de Extranjeros con } \\
\text { Autorización de Estancia por } \\
\text { Estudios en Vigor. }\end{array}$ \\
\hline & $\begin{array}{l}\text { Residencia de larga } \\
\text { duración }\end{array}$ & $\begin{array}{l}\text { Tasa de residencia de larga duración }=\left(\mathrm{N} .^{\circ} \text { de }\right. \\
\text { personas con autorizaciones de residencia de } \\
\text { larga duración / N. } .^{\circ} \text { de personas en el Régimen } \\
\text { General })^{\star} 100 .\end{array}$ & $\begin{array}{l}\text { Registro de Extranjeros con } \\
\text { Certificado de Registro o Tarjeta } \\
\text { de Residencia en Vigor. }\end{array}$ \\
\hline & $\begin{array}{l}\text { Nacionalización por } \\
\text { residencia }\end{array}$ & $\begin{array}{l}\text { Tasa acumulativa de naturalización }=\left(\mathrm{N} .^{\circ} \text { de }\right. \\
\text { personas naturalizadas españolas en el periodo } \\
\mathrm{X}-\mathrm{Y} / \mathrm{N} .^{0} \text { de personas naturalizadas en el periodo } \\
\mathrm{X}-\mathrm{Y}+\mathrm{N} .^{\circ} \text { de personas extranjeras empadrona- } \\
\text { das en el año } \mathrm{Y}+1){ }^{*} 100 .\end{array}$ & $\begin{array}{l}\text { Registro de Concesiones } \\
\text { de Nacionalidad Española por } \\
\text { Residencia. }\end{array}$ \\
\hline & Sufragio activo & $\begin{array}{l}\text { Tasa de inscripción en el CERE }=\left(\mathrm{N} .{ }^{\circ} \text { de per- }\right. \\
\text { sonas extranjeras inscritas en el CERE / N. }{ }^{\circ} \text { de } \\
\text { comunicaciones enviadas por la Oficina del } \\
\text { Censo Electoral a personas extranjeras con } \\
\text { derecho a voto en España) }{ }^{*} 100 .\end{array}$ & $\begin{array}{l}\text { Banco de Datos de Personas } \\
\text { Electas a Órganos Representati- } \\
\text { vos Locales. }\end{array}$ \\
\hline & Sufragio pasivo & $\begin{array}{l}\text { Tasa de personas electas }=\left(N .^{\circ} \text { de personas }\right. \\
\text { electas en las elecciones locales de un año } X \text {, } \\
\text { según su lugar de nacimiento } / N .^{\circ} \text { de personas } \\
\text { empadronadas a } 1 / 1 \text { del año } X \text {, según su lugar } \\
\text { de nacimiento })^{*} 1000 \text {. }\end{array}$ & Censo Electoral. \\
\hline & Escolaridad infantil & $\begin{array}{l}\text { Tasa bruta de escolaridad = (Alumnado matri- } \\
\text { culado en infantil, en el curso X / Población en } \\
\text { edad teórica de cursar infantil, empadronada al } \\
\text { inicio del } 2^{\circ} \text { año del curso X) }{ }^{*} 100 \text {. }\end{array}$ & $\begin{array}{l}\text { Sistema Estatal de Indicadores } \\
\text { de la Educación. }\end{array}$ \\
\hline & $\begin{array}{l}\text { Escolaridad postobli- } \\
\text { gatoria }\end{array}$ & $\begin{array}{l}\text { Tasa bruta de escolaridad = (Alumnado matri- } \\
\text { culado en secundaria no obligatoria, en el curso } \\
\text { X / Población en edad teórica de cursar secun- } \\
\text { daria no obligatoria, empadronada al inicio del } \\
2 .^{\circ} \text { año del curso X) }{ }^{*} 100 \text {. }\end{array}$ & $\begin{array}{l}\text { Sistema Estatal de Indicadores } \\
\text { de la Educación. }\end{array}$ \\
\hline
\end{tabular}

Fuente: elaboración propia. 